Reprod. Nutr. Dévelop., 1980, 20 (5 B), 1707-1715.

\title{
Influence de l'aliment et de la microfiore sur la teneur en sucres aminés des contenus digestifs et des fèces chez le rat, l'agneau et le veau préruminant
}

\author{
par Etiennette COMBE, P. PATUREAU-MIRAND, G. BAYLE, R. PION \\ Laboratoire d'éfude du Métabolisme Azoté, INRA, \\ Theix, St-Genès-Champanelle 63110 Beaumont, France.
}

Summary. Effect of diet and microflora on amino sugars in the digestive contents and faeces of rats, lambs and preruminant calves.

Glucosamine $\left(\mathrm{Glc} \mathrm{NH}_{2}\right)$ and galactosamine $\left(\mathrm{Gal} \mathrm{NH} \mathrm{NH}_{2}\right)$ were determined by an automatized chromatographic method in samples from digestive contents and faeces. Germfree lambs and rats as well as conventional preruminant calves, lambs and rats were used in the experiments. The rats were fed an irradiated casein diet, the lambs UHT milk and the preruminant calves various diets (table 1). Concentrations of Glc $\mathrm{NH}_{2}$ and of $\mathrm{Gal} \mathrm{NH}_{2}$ reached $6 \mathrm{~g} / 16 \mathrm{~g}$ of $\mathrm{N}$ in the caecal contents and the faeces of germfree rats (table 2). Concentrations of $\mathrm{Gal} \mathrm{NH} \mathrm{NH}_{2}$ were 7 to 12 times lower and those of Glc NH 4 times lower in conventional rats. In lambs, the amino sugar concentrations (table 3 ) found in the stomach contents resembled those supplied by the milk. In contrast, in the large intestine and the faeces, the concentration of amino sugars was very high. In conventional lambs, those concentrations were 8 to 4 times lower. For ruminant calves, the concentrations of amino sugars (table 4) were greater in the ileal contents than in the faeces. They reached that of meconium when the calves were fed milk. The lowest values were observed in the faeces of calves fed soya, colostrum or fish diets which are less digestible than milk. In germfree animals, the hexosamines were endogenous. In conventional animals, bacterial metabolism led to an increase in faecal Glc $\mathrm{NH}_{2} / \mathrm{Gal} \mathrm{NH}_{2}$. In the calves fed soya or fish diets, the hexosamine content in the faeces was low owing to the dilution of bacterial and endogenous mucoproteins by the undigested dietary proteins.

De nombreux auteurs (Bella et Kim, 1972 ; De Caro et al., 1977 ; Goso et Hotta, 1977 ; Hill, Reynolds et Hill, 1977 ; Slomiany et Slomiany, 1978) se sont intéressés à la composition en acides aminés et en sucres aminés des contenus digestifs ayant subi différents modes de purification ou de fractionnement. lls ont montré que les mucoprotéines digestives présentaient une forte proportion de thréonine et de sérine et des proportions de glucosamine et de galactosamine élevées mais variables entre elles selon le degré de purification et l'origine des composés étudiés. Nous avons donc complété l'analyse de la composition en acides aminés des contenus digestifs et des fèces obtenue chez le rat, chez l'agneau axéniques ou holoxéniques et chez le veau soumis à divers régimes par la détermination des sucres aminés présents dans ces échantillons (Pion ef al., 1978). 


\section{Conditions expérimentales.}

1. Rats. - Nous avons utilisé de jeunes rats mâles sevrés de race Sprague Dawley en cages individuelles munies d'un sol grillagé. Le protocole expérimental a nécessité une mise au point particulière pour certains aspects. Les 8 rats axéniques OFA ont été répartis dans 2 isolateurs contenant chacun 4 cages. Les 5 rats holoxéniques ont été placés dans leur cage à l'air libre dans la pièce contenant les isolateurs maintenue à $21^{\circ} \mathrm{C} \pm 1$ après avoir vérifié par ailleurs que l'effet « isolateur » n'intervenait pas sur les quantités d'aliment ingérées. Le régime contenait $15 \mathrm{p} .100$ de caséine (obtenue par précipitation acide) et 64 p. 100 d'amidon. It était supplémenté en méthionine, arginine et tryptophane ainsi qu'en vitamines et minéraux de façon à couvrir les besoins du rat en croissance. Le mélange réalisé sous forme de poudre a été conditionné sous vide en doses journalières individuelles de $20 \mathrm{~g}$, sous double emballage plastique, pour être stérilisé par irradiation à 4,5 Mrads. La ration journalière était présentée aux rats dans des ramequins en verre, sous forme semi-liquide obtenue après avoir ajouté $20 \mathrm{ml}$ d'eau ef la dose adéquate du mélange d'huile vitaminée. Pendant 2 semaines consécutives les consommations et les croissances individuelles ont été mesurées ; au cours de la troisième semaine, les fèces ont été recueillies pendant 5 jours grâce à un dispositif de séparation de l'urine et des fèces puis lyophilisées. Au bout de la période expérimentale, la croissance moyenne des rats élait de $5,5 \mathrm{~g} /$ jour pour une ingestion moyenne de $15 \mathrm{~g} \mathrm{MS/jour} \mathrm{avec} \mathrm{une} \mathrm{excrétion} \mathrm{fécale} \mathrm{de}$ $1,3 \mathrm{~g} \mathrm{MS/jour.} \mathrm{Ensuite} \mathrm{les} \mathrm{animaux} \mathrm{ont} \mathrm{été} \mathrm{sacrifiés} \mathrm{et} \mathrm{les} \mathrm{contenus} \mathrm{des} \mathrm{cæcums} \mathrm{préle-}$ vés congelés et lyophilisés. Les analyses ont été effectuées sur les mélanges de contenus de cæcums et les fèces provenant respectivement des rats axéniques de chacun des isolateurs ( $\mathrm{Ax}$. A et Ax. B) ef des rats holoxéniques (Holox.).

2. Agneaux. - Les agneaux holoxéniques et axéniques ont été élevés selon la procédure expérimentale déjà décrite (Riou et al., 1977). Ils recevaient du lait de vache stérilisé par ultra haute température (UHT). Leurs croissances étaient respectivement de 180 et $260 \mathrm{~g} /$ jour pour une ingestion moyenne de 215 et $315 \mathrm{~g} \mathrm{MS} /$ jour avec une excrétion fécale de 8,34 et $6,07 \mathrm{~g} \mathrm{MS/jour} \mathrm{(Combe,} \mathrm{1976).} \mathrm{A} \mathrm{la} \mathrm{fin} \mathrm{de} \mathrm{la} \mathrm{période} \mathrm{de}$ récolte des fèces ( 5 jours en fin de deuxième semaine), 2 des agneaux axéniques ( $C$ et $D)$ ont été sacrifiés et les contenus digestifs prélevés, congelés et lyophilisés. Les analyses ont été faites sur l'aliment, le mélange des fèces des agneaux holoxéniques, les fèces ef le mélange des contenus des réservoirs gastriques (RG) et de gros intestin (GI) des agneaux axéniques.

3. Veaux. - Les échantillons de contenus d'iléon et de fèces ont éfé recueillis au cours de différentes études de la digestion chez le veau réalisées à l'INRA par le laboratoire de Zootechnie de Rennes, celui de la Digestion des Ruminants de Theix, et par nous-mêmes. Les conditions expérimentales, la composition des aliments ef les quantités ingérées sont résumées dans le tableau 1. Dans le cas des aliments Poisson, Soja, Féverole et Levures, seule une fraction des protéines est fournie par ces produits, le reste étant apporté par une poudre de lactosérum et/ou de lait écrémé. L'aliment Colostrum est préparé par le mélange du produit des 4 premières traites de plusieurs vaches. 
TABLEAU 1

Conditions ef composition des aliments des expériences sur veoux préruminants

\begin{tabular}{|c|c|c|c|c|c|c|c|c|c|}
\hline \multicolumn{3}{|c|}{ Echantillons } & \multicolumn{4}{|c|}{ Conditions expérimentales } & \multicolumn{3}{|c|}{$\begin{array}{l}\text { Composition de l'aliment } \\
\text { (p. } 100 \mathrm{MS} \text { ) }\end{array}$} \\
\hline & Aliment & $\begin{array}{l}\text { No } \\
\text { expér. }\end{array}$ & $\begin{array}{c}\text { Nbre } \\
\text { ani- } \\
\text { maux }\end{array}$ & $\begin{array}{l}\text { Nbre jours } \\
\text { de pré- } \\
\text { lèvements }\end{array}$ & $\begin{array}{c}\text { MS } \\
\text { ingérée } \\
\text { g/jour }\end{array}$ & $\begin{array}{c}\text { Poids } \\
\text { vif } \\
(\mathrm{kg})\end{array}$ & $\begin{array}{c}\text { Matières } \\
\text { azotées } \\
\text { totales }\end{array}$ & $\begin{array}{l}\text { Matières } \\
\text { grasses }\end{array}$ & $\begin{array}{c}\text { MAE (1) } \\
\text { P. } 100 \\
\text { MAT }\end{array}$ \\
\hline \multirow{3}{*}{$\begin{array}{l}\text { Contenus } \\
\text { d'iléon }\end{array}$} & Lait & $\begin{array}{l}1 \\
2 \\
3\end{array}$ & $\begin{array}{l}3 \\
2 \\
3\end{array}$ & $\begin{array}{l}3 \\
1 \\
1\end{array}$ & $\begin{array}{l}1505 \\
1298 \\
1528\end{array}$ & $\begin{array}{l}98 \\
77 \\
94\end{array}$ & $\begin{array}{l}24,6 \\
25,3 \\
25,3\end{array}$ & $\begin{array}{l}20,5 \\
20,2 \\
20,2\end{array}$ & $\begin{array}{l}100 \\
100 \\
100\end{array}$ \\
\hline & Soja & & 2 & 3 & 1713 & 120 & 25,9 & 19 & 74 \\
\hline & Féverole & $\begin{array}{l}1 \\
2 \\
3\end{array}$ & $\begin{array}{l}1 \\
2 \\
1\end{array}$ & $\begin{array}{l}3 \\
3 \\
3\end{array}$ & $\begin{array}{l}1140 \\
1140 \\
1131\end{array}$ & $\begin{array}{l}60 \\
60 \\
59\end{array}$ & $\begin{array}{l}25,8 \\
25,6 \\
25,7\end{array}$ & $\begin{array}{l}21,2 \\
21,1 \\
22,1\end{array}$ & $\begin{array}{l}41 \\
41 \\
41\end{array}$ \\
\hline \multirow{6}{*}{ Fèces } & Lait & $\begin{array}{l}1 \\
2 \\
3\end{array}$ & $\begin{array}{l}3 \\
2 \\
2\end{array}$ & $\begin{array}{r}5 \\
11 \\
11\end{array}$ & $\begin{array}{l}2410 \\
1769 \\
2010\end{array}$ & $\begin{array}{r}127 \\
98 \\
145\end{array}$ & $\begin{array}{l}24,8 \\
25,3 \\
25,3\end{array}$ & $\begin{array}{l}20,5 \\
20,2 \\
20,2\end{array}$ & $\begin{array}{l}100 \\
100 \\
100\end{array}$ \\
\hline & Soja & & 3 & 11 & 1714 & 81 & 24,8 & 20,0 & 78,0 \\
\hline & Féverole & $\begin{array}{l}1 \\
2 \\
3 \\
4 \\
5\end{array}$ & $\begin{array}{l}3 \\
4 \\
5 \\
4 \\
3\end{array}$ & $\begin{array}{l}11 \\
10 \\
10 \\
10 \\
11\end{array}$ & $\begin{array}{l}1443 \\
1591 \\
1745 \\
1811 \\
1483\end{array}$ & $\begin{array}{r}78 \\
101 \\
108 \\
114 \\
82\end{array}$ & $\begin{array}{l}24,2 \\
25,8 \\
25,6 \\
25,7 \\
26,2\end{array}$ & $\begin{array}{l}20,6 \\
21,2 \\
21,1 \\
22,1 \\
18,1\end{array}$ & $\begin{array}{l}73 \\
41 \\
41 \\
41 \\
50\end{array}$ \\
\hline & Colostrum & $\begin{array}{l}1 \\
2\end{array}$ & $\begin{array}{l}3 \\
3\end{array}$ & $\begin{array}{l}5 \\
5\end{array}$ & $\begin{array}{l}914 \\
787\end{array}$ & $\begin{array}{l}50 \\
41\end{array}$ & $\begin{array}{l}39,3 \\
38,8\end{array}$ & $\begin{array}{l}27,7 \\
26,6\end{array}$ & $\begin{array}{l}100 \\
100\end{array}$ \\
\hline & Poisson & & 3 & 11 & 1421 & 74 & 26,6 & 20,0 & 81,0 \\
\hline & Levures & $\begin{array}{l}1 \\
2 \\
3\end{array}$ & $\begin{array}{l}3 \\
2 \\
3\end{array}$ & $\begin{array}{r}6 \\
11 \\
11\end{array}$ & $\begin{array}{l}1956 \\
1805 \\
1383\end{array}$ & $\begin{array}{r}101 \\
103 \\
73\end{array}$ & $\begin{array}{l}24,2 \\
25,2 \\
23,7\end{array}$ & $\begin{array}{l}18,0 \\
20,2 \\
18,0\end{array}$ & $\begin{array}{l}48 \\
40 \\
75\end{array}$ \\
\hline Méconium & & & 4 & 1 & - & 38 & - & - & - \\
\hline
\end{tabular}

(1) Matières azotées apportées dans le régime par les produits étudiés (MAE).

Les contenus d'iléon ont été prélevés à la fin de l'iléon à l'aide d'une canule simple (expérience Lait 1, Soja) ou réentrante (expérience Lait 2, 3 et Féverole) placée peu avant la valvule iléocæcale. Les fèces étudiées ont été récoltées sur des veaux différents des précédents mais qui recevaient le même régime.

La détermination de la composition en sucres aminés est réalisée sur chacun des échantillons moyens constitués du mélange des fèces ou contenus d'iléon des veaux participant à une même expérience. 
4. Méthodes de dosage. - Les sucres aminés sont libérés par hydrolyse acide ménagée par $\mathrm{HCl} 4 \mathrm{~N}$ à $100^{\circ} \mathrm{C}$ pendant $2 \mathrm{~h}$. Ils sont ensuite séparés par chromatographie échangeuse de cations (résine BioRad Laboratories Aminex 44). L'élution est réalisée par un gradient $(\mathrm{pH}$, concentration en $\mathrm{Na}$ ) réalisé au moyen de tampons citrate (Monsigny, 1969).

Ils sont dosés par la réaction de Elson-Morgan au moyen d'une chaîne d'analyse automatique par la technique de Monsigny (1969). Le caractère quantitatif de l'acétylation ef de la réaction colorée est contrôlé grâce au dosage d'une gamme étalon de glucosamine et de $\mathrm{N}$ acétyl-glucosamine au début et à la fin de chaque chromatogramme.

\section{Résultats.}

1. Rats (tabl. 2). - Les teneurs en sucres aminés de l'aliment sont pratiquement nulles. Dans les contenus de cæcum elles sont élevées dans le cas des rats axéniques. Les teneurs en glucosamine varient entre 5,9 à $7,3 \mathrm{~g} / 16 \mathrm{~g} \mathrm{~N}$, ef les teneurs en galactosamine entre 6,4 et $7 \mathrm{~g} / 16 \mathrm{~g} \mathrm{~N}$ selon les lots. Elles n'atteignent que 0,9 et 1,6 g pour

TABLEAU 2

Influence de la microflore sur les teneurs en sucres aminés $(\mathrm{g} / 16 \mathrm{~g} \mathrm{~N})$ dans le cæcum ef les fèces des rots

\begin{tabular}{|c|c|c|c|c|c|}
\hline Animaux & Echantillons & $\mathrm{N}$ p. $100 \mathrm{MS}$ & Glc. $\mathrm{NH}_{2}$ & $\mathrm{Gal} . \mathrm{NH}_{2}$ & $\mathrm{Glc} / \mathrm{Gal}$ \\
\hline & Aliment & 2,51 & 0 & 0 & 一 \\
\hline $\begin{array}{l}\text { Ax. A } \ldots \ldots \ldots \ldots \\
\text { Ax. B } \ldots \ldots \cdots \cdots \\
\text { Holox........ }\end{array}$ & Contenu de cæcum & $\begin{array}{l}2,70 \\
2,70 \\
3,38\end{array}$ & $\begin{array}{l}7,3 \\
5,9 \\
1,6\end{array}$ & $\begin{array}{l}7,0 \\
6,4 \\
0,9\end{array}$ & $\begin{array}{l}1,0 \\
0,9 \\
1,7\end{array}$ \\
\hline $\begin{array}{l}\text { Ax. A } \ldots \ldots \ldots \ldots \\
\text { Ax. B } \ldots \ldots \ldots \ldots \\
\text { Holox......... }\end{array}$ & Fèces & $\begin{array}{l}3,26 \\
3,39 \\
4,11\end{array}$ & $\begin{array}{l}6,3 \\
5,3 \\
1,4\end{array}$ & $\begin{array}{l}7,1 \\
6,5 \\
0,6\end{array}$ & $\begin{array}{l}0,9 \\
0,8 \\
2,6\end{array}$ \\
\hline
\end{tabular}

$16 \mathrm{~g}$ d'azote respectivement pour la galactosamine et la glucosamine dans les contenus de cæcum des rats holoxéniques. Le rapport glucosamine/galactosamine est toujours plus élevé chez les rats holoxéniques que chez les axéniques. Dans les fèces, les teneurs en sucres aminés sont également plus élevées chez les axéniques que chez les holoxéniques. Le rapport glucosamine/galactosamine est également plus élevé chez les holoxéniques que chez les axéniques. A la suite du transit dans le côlon et le rectum des rats axéniques la teneur en azote des digesta augmente mais les composés azotés ont tendance à se trouver légèrement appauvris en glucosamine et très légèrement enrichis en galactosamine. Dans le cas des rats holoxéniques, où s'ajoutent prolifération microbienne ef action de la microflore sur le métabolisme de l'épithélium et des autres tissus digestifs (Combe ef Debras, 1976), le transit dans le côlon ef le rectum se traduit aussi par une augmentation de la teneur en matières azotées des digesta dont les teneurs en glucosamine sont réduites de 10 p. 100 et celles de galactosamine de 45 p. 100. 
2. Agneaux (tabl. 3). - Les teneurs en glucosamine ef galactosamine des composés azotés du lait ef des contenus des réservoirs gastriques des agneaux axéniques sont faibles $(0,4 \mathrm{à} 0,6 \mathrm{~g} / 16 \mathrm{~g} \mathrm{~N})$ et peu différentes entre elles. Par contre les teneurs en sucres aminés des composés azotés provenant du gros intestin des agneaux axéni-

TABLEAU 3

Influence de la microflore sur les teneurs en sucres aminés $(\mathrm{g} / 16 \mathrm{~g} \mathrm{~N})$ des contenus digestifs et des fèces des agneaux

\begin{tabular}{|c|c|c|c|c|c|}
\hline Animaux & Echantillons & N p. $100 \mathrm{MS}$ & Gic. $\mathrm{NH}_{2}$ & Gal. $\mathrm{NH}_{2}$ & $\mathrm{Glc} / \mathrm{Gal}$ \\
\hline & Aliment & 4,61 & 0,6 & 0,4 & 1,5 \\
\hline $\begin{array}{l}\text { Ax. } C+D \ldots \ldots \\
\text { Ax. } C+D, \ldots \cdots\end{array}$ & $\begin{array}{l}\text { R. G. } \\
\text { G. I. }\end{array}$ & $\begin{array}{l}5,67 \\
6,35\end{array}$ & $\begin{array}{l}0,6 \\
7,8\end{array}$ & $\begin{array}{l}0,5 \\
8,2\end{array}$ & $\begin{array}{l}1,2 \\
0,9\end{array}$ \\
\hline $\begin{array}{l}\text { Ax. C } \ldots \ldots \cdots \cdots \\
\text { Ax. D } \ldots \ldots \cdots \cdots \cdots \\
\text { Holox. } \ldots \ldots \ldots \ldots\end{array}$ & Fèces & $\begin{array}{l}6,42 \\
4,87 \\
7,52\end{array}$ & $\begin{array}{l}7,4 \\
8,6 \\
2,3\end{array}$ & $\begin{array}{l}8,8 \\
7,0 \\
1,2\end{array}$ & $\begin{array}{l}0,8 \\
1,2 \\
1,9\end{array}$ \\
\hline
\end{tabular}

ques sont élevées $(7,8 \mathrm{~g}$ et $8,2 \mathrm{~g} / 16 \mathrm{~g} \mathrm{~N}$ pour la glucosamine et la galactosamine, respectivement). Au cours du passage dans l'intestin grêle, les contenus digestifs ont donc été enrichis en sucres aminés et particulièrement en galactosamine. Les teneurs en sucres aminés présentes dans les fèces des 2 agneaux axéniques sont toujours élevées : les teneurs en glucosamine et en galactosamine varient entre 7 et près de $8 \mathrm{~g} / 16 \mathrm{~g} \mathrm{~N}$. La différence entre les teneurs observées dans les fèces provenant des deux agneaux étudiés souligne la variabilité individuelle.

Les teneurs en sucres aminés des fèces des agneaux holoxéniques atteignent $2,3 \mathrm{~g}$ et $1,2 \mathrm{~g} / 16 \mathrm{~g} \mathrm{~N}$ pour la glucosamine et la galactosamine, respectivement. Elles sont nettement inférieures à celles décrites dans les fèces ou les contenus de gros intestin des agneaux axéniques. La teneur en glucosamine est près de 2 fois plus élevée que celle en galactosamine. Cette différence importante a été notée également dans les fèces et le cæcum des rats holoxéniques.

3. Veaux (tabl. 4). - Les différents aliments utilisés par les veaux ont des teneurs variables en sucres aminés : le lait de vache contient peu de glucosamine et galactosamine $(0,6$ et $0,4 \mathrm{~g} / 16 \mathrm{~g} \mathrm{~N}$ respectivement) ; le colostrum contient $0,3 \mathrm{~g}$ de galactosamine mais il est plus riche en glucosamine $(1,0 \mathrm{~g})$; les levures sont particulièrement riches en glucosamine $(1,5 \mathrm{~g})$ et pauvres en galactosamine (teneurs inférieures à $0,15 \mathrm{~g})$. Quant aux aliments Poisson, Soja, et Féverole, leurs teneurs en glucosamine et galactosamine étaient inférieures ou égales à celles du lait.

Les teneurs en glucosamine sont plus élevées dans les contenus d'iléon que dans les fèces ; cela est encore plus net pour la galactosamine. Les teneurs en sucres aminés des contenus d'iléon des veaux nourris avec les aliments Lait sont les plus élevées et sont proches de celles du méconium de veau ainsi que des contenus de gros intestin ef des fèces d'agneau axénique. Les contenus d'iléon des veaux recevant l'aliment Féverole sont les moins riches en azote et en glucosamine ; ceux des veaux nourris avec l'aliment Soja ont le rapport glucosamine/galactosamine le plus élevé. 
TABLEAU 4

Teneurs en sucres aminés des contenus d'iléon et des fèces de veaux préruminants $(\mathrm{g} / 16 \mathrm{~g} \mathrm{~N}$ )

\begin{tabular}{|c|c|c|c|c|c|c|}
\hline \multicolumn{3}{|c|}{ Echantillons } & \multicolumn{4}{|c|}{ Composition des digesta } \\
\hline & Aliment & $\begin{array}{c}\text { No } \\
\text { expér. }\end{array}$ & N p. $100 \mathrm{MS}$ & Glc. $\mathrm{NH}_{2}$ & $\mathrm{Gal} . \mathrm{NH}_{2}$ & Glc/Gal \\
\hline \multirow{3}{*}{$\begin{array}{c}\text { Contenus } \\
\text { d'iléon }\end{array}$} & Lait & $\begin{array}{l}M\left({ }^{1}\right) \\
1 \\
2 \\
3\end{array}$ & $\begin{array}{l}3,07 \\
3,15 \\
3,02 \\
3,01\end{array}$ & $\begin{array}{l}\mathbf{7 , 8} \\
\mathbf{7 , 1} \\
7,7 \\
\mathbf{8 , 5}\end{array}$ & $\begin{array}{l}6,6 \\
5,2 \\
7,2 \\
7,5\end{array}$ & $\begin{array}{l}1,2 \\
1,4 \\
1,1 \\
1,1\end{array}$ \\
\hline & Soja & & 3,55 & 4,9 & 2,9 & 1,7 \\
\hline & Féverole & $\begin{array}{l}M\left({ }^{1}\right) \\
1 \\
2 \\
3\end{array}$ & $\begin{array}{l}2,34 \\
2,84 \\
2,19 \\
1,98\end{array}$ & $\begin{array}{l}4,0 \\
4,4 \\
4,3 \\
3,4\end{array}$ & $\begin{array}{l}3,3 \\
4,1 \\
3,5 \\
2,4\end{array}$ & $\begin{array}{l}1,2 \\
1,1 \\
1,2 \\
1,4\end{array}$ \\
\hline \multirow{6}{*}{ Fèces } & Lait & $\begin{array}{l}M(1) \\
1 \\
2 \\
3\end{array}$ & $\begin{array}{l}3,90 \\
4,60 \\
3,04 \\
3,72\end{array}$ & $\begin{array}{l}3,0 \\
2,8 \\
2,4 \\
3,80\end{array}$ & $\begin{array}{l}2,2 \\
2,5 \\
1,5 \\
2,3\end{array}$ & $\begin{array}{l}1,4 \\
1,1 \\
1,6 \\
1,7\end{array}$ \\
\hline & Soja & & 4,70 & 1,3 & 0,7 & 1,9 \\
\hline & Féverole & $\begin{array}{l}M(1) \\
1 \\
2 \\
3 \\
4 \\
5\end{array}$ & $\begin{array}{l}4,28 \\
4,39 \\
4,94 \\
3,99 \\
3,81 \\
4,38\end{array}$ & $\begin{array}{l}3,1 \\
2,8 \\
2,1 \\
3,7 \\
4,1 \\
1,4\end{array}$ & $\begin{array}{l}2,0 \\
1,6 \\
1,5 \\
2,2 \\
2,7 \\
1,3\end{array}$ & $\begin{array}{l}1,6 \\
1,8 \\
1,4 \\
1,7 \\
1,5 \\
1,1\end{array}$ \\
\hline & Colostrum & $\begin{array}{l}M(1) \\
1 \\
2\end{array}$ & $\begin{array}{r}10,02 \\
11,44 \\
8,60\end{array}$ & $\begin{array}{l}1,4 \\
1,2 \\
1,6\end{array}$ & $\begin{array}{l}0,7 \\
0,4 \\
0,9\end{array}$ & $\begin{array}{l}2,4 \\
3,0 \\
1,8\end{array}$ \\
\hline & Poisson & & 6,70 & 1,2 & 0,7 & 1,7 \\
\hline & Levures & $\begin{array}{l}M\left({ }^{1}\right) \\
1 \\
2 \\
3\end{array}$ & $\begin{array}{l}5,25 \\
5,07 \\
5,04 \\
5,58\end{array}$ & $\begin{array}{l}4,0 \\
3,5 \\
4,1 \\
4,3\end{array}$ & $\begin{array}{l}1,0 \\
0,8 \\
1,0 \\
1,3\end{array}$ & $\begin{array}{l}3,9 \\
4,4 \\
4,1 \\
3,3\end{array}$ \\
\hline Méconium & & & 6,72 & 7,8 & 6,9 & 1,1 \\
\hline
\end{tabular}

(1) Moyenne pondérée en fonction du nombre d'animaux.

Les teneurs en glucosamine ainsi que celles en galactosamine sont les plus fortes dans les fèces des veaux qui consomment les aliments Lait ou Féverole; seule la teneur en glucosamine est élevée dans les fèces des veaux nourris avec les aliments Levures. Les teneurs en glucosamine ainsi que celles en galactosamine sont relativement faibles et comparables dans les fèces de veaux recevant les aliments Soja, Colostrum et Poisson. Le rapport glucosamine/galactosamine est plus élevé dans les fèces que dans les contenus d'iléon ; il est particulièrement fort dans les fèces des veaux recevant l'aliment Levures. 


\section{Discussion.}

Influence de la microflore. - Comme le régime donné à nos rats ne comportait pas de sucres aminés décelables, les hexosamines présentes dans les digesta ont une origine endogène indiscutable. Lindstedt, Lindstedt et Gustafson (1965) avaient déjà montré que la teneur en sucres aminés des digesta était 5 fois plus élevée dans le cas des rats axéniques que dans celui des holoxéniques. Les faibles teneurs en sucres aminés observées dans le cas des rats holoxéniques proviennent de destructions et de remaniements imputables aux métabolismes de la microflore. Ceux-ci se traduisent dans les fèces par une augmentation du rapport glucosamine/galactosamine provenant du développement des bactéries dont les parois sont peu digestibles et riches en glucosamine (Ghuysen, Strominger et Tipper, 1968). De même dans le cas des agneaux holoxéniques les teneurs en sucres aminés des fèces sont faibles et le rapport glucosamine/galactosamine élevé quand il est comparé aux résultats obtenus dans le cas des agneaux axéniques.

Chez les agneaux axéniques, l'addition aux aliments, des sécrétions salivaire et gastrique modifie peu la teneur en sucres aminés des composés azotés dont la proportion est cependant plus élevée dans les contenus stomacaux que dans l'aliment. L'accroissement important des teneurs en glucosamine et en galactosamine dans les composés azotés digestifs entre les réservoirs gastriques et le gros intestin provient des sécrétions digestives et de la muqueuse intestinale qui sont très riches en sucres aminés (Spiro, 1970).

Influence de l'espèce. - Les teneurs en sucres aminés des fèces des agneaux et des veaux nourris au lait semblent plus élevées que celles des fèces des rats. Quelle que soit l'espèce, la présence de microflore se traduit par une baisse des teneurs en sucres aminés et surtout en galactosamine, à la suife d'une destruction d'une partie importante des mucoprotéines endogènes dans le gros intestin. Chez le rat et chez l'agneau ce phénomène est mis en évidence par les différences de teneurs entre les contenus de cæcum ef les fèces. Chez le veau une telle dégradation peut être déduite de la comparaison de la composition en sucres aminés des contenus d'iléon de veaux nourris à l'aide d'aliments très digestibles (Lait) à celle de leurs fèces ; en effet, la population microbienne de la fin de l'iléon chez le veau est environ 10 fois moins importante que dans les fèces (Contrepois, 1973). Cela est confirmé par l'étude des teneurs en acides aminés de ces digesta (Patureau-Mirand et al., 1977) : dans les contenus d'iléon de ces animaux, les mucoprotéines intestinales constituent la majeure partie des protéines, alors que dans les fèces ce sont les protéines bactériennes (Guilloteau et al., 1980).

Influence de l'aliment. - La faible teneur en sucres aminés des contenus de fin d'iléon des veaux recevant les aliments Soja ef Féverole, comparée à celle des veaux nourris au lait, résulte en partie de la dilution des mucoprotéines intestinales par des protéines indigérées de l'aliment. La composition en acides aminés des mêmes échantillons indique d'ailleurs leur présence à ce niveau en proportion apparemment plus importante que dans le cas des aliments Lait (Toullec et al., 1980).

Les concentrations élevées en galactosamine des fèces des veaux qui consom- 
ment les aliments Lait et Féverole s'expliquent par la présence en quantités non négligeables de mucoprotéines intestinales à ce niveau (Guilloteau ef al., 1980 ; Toullec ef al., 1980). Les fortes teneurs en glucosamine des fèces des veaux nourris avec les aliments Levures semblent indiquer que les parois des levures, riches en ce composé (Sentandreu ef Northcote, 1968) ne sont que partiellement digérées (Besle, Lassalas et Thivend, 1980) et contribuent à accroître la teneur en glucosamine des fèces. II pourrait en être de même d'une partie de la glucosamine des fèces des veaux âgés de 8 à 13 jours nourris au colostrum ; elle proviendrait de certaines protéines du colostrum riches en glucosamine (immunoglobulines notamment), qui ne paraissent qu'incomplètement digérées à cet âge, comme en témoigne la composition en acides aminés des fèces des veaux recevant cet aliment (Pion ef al., 1978).

\section{Journées Ingestion-Digestion-Absorption de l'Association française de Nutrition, Paris, 15-16 novembre 1979.}

Remerciements. - Nous remercions le Laboratoire de Microbiologie (INRA-Theix) qui nous a donné toutes les facilités pour assurer l'entretien des agneaux axéniques, ainsi que MM. Besle, Guilloteau, Thivend, Toullec et Troccon qui nous ont fourni la plupart des échantillons de digesta de veaux préruminants.

\section{Références}

BELLA A., KIM Y. S., 1972. Rat small intestinal mucus : isolation and characterization of a water soluble mucin fraction. Arch. Biochem. Biophys., 150, 679-689.

BESLE J. M., LASSALAS B., THIVEND P., 1980. Digestion des glucides de la levure d'alcanes par le veau préruminant. Rep. Nutr. Dév., 20, $1401-1414$.

COMBE E., 1976. Influence de la microflore intestinale sur la composition en acides aminés des fèces des agneaux. C. R. Soc. Biol., 170, 787-793.

COMBE E., DEBRAS E., 1976. Influence de la microflore sur le métabolisme de la L-thréonine au niveau du tube digestif du rat. $C$. R. Soc. Biol., 170, 368-374.

CONTREPOIS M., 1973. La microflore du tube digestif du jeune veau préruminant. Dénombrement de quelques groupes bactériens à différents niveaux du tube digestif. Ann. Rech. vét., 4, 161-170.

DE CARO A., FIGARELLA C., AMIC J., MICHEL R., GUYO., 1977. Human pancreatic lipase : a glycoprotein. Bioch. bioph. Acta, 490, 411-419.

GHUYSEN J. M., STROMINGER J. L., TIPPER D. J., 1968. Bacterial cell wall, in Comprehensive biochemistry, 26A, 53-104. M. FLORKIN ef E. M. STOTZ, Amsterdam.

GOSO K., HOTTA K., 1977. Glycoprotein isolated from human gastric juice. Biochem. J., 163, 169-172.

GUILLOTEAU P., PATUREAU-MIRAND P., TOULLEC R., PRUGNAUD J., 1980. Digestion of milk protein and methanol-grown bacteria protein in the preruminant calf. II. Amino acid composition of ileal digesta and faeces and blood levels of free amino acids. Reprod. Nutr. Dévelop., 20, 615-629.

HILL M. O. Jr, REYNOLDS J. A., HILL R. L., 1977. Purification, composition, molecular weight and subunit structure of ovine submaxillary mucus. J. biol. Chem., 252, 3791-3798.

LINDSTEDT G., LINDSTEDT S., GUSTAFSON B. E., 1965. Mucus in intestinal contents of germfree rats. J. exp. Med., 121, $201-213$.

MONSIGNY M., 1969. Ełudes sur les glycoproteines. Bull. Soc. Chim. biol., 51, 1263-1269.

PATUREAU-MIRAND P., TOULLEC R., GUILLOTEAU P., PION R., 1977. Influence de la nature des protéines alimentaires sur la composition en acides aminés des fèces du veau préruminant. Ann. Biol. anim. Bioch. Biophys., 17, 71-83.

PION R., PATUREAU-MIRAND P., COMBE E., MENDES PEREIRA E., 1978. Effects of some factors on digestive utilisation of amino acids from feeds. Congr. int. Nutr., Rio de Janeiro. 
RIOU Y., GOUET Ph., DUBOURGUIER H. C., CONTREPOIS M., DARDILLAT C., LEFAIVRE J., 1977. Techniques for obtaining, fistulization and rearing of axenic or gnotobiotic lambs, kids and calves. Ann. Rech, vét., 8, 13-24.

SENTANDREU R., NORTHCOTE D. H., 1968. The structure of a glycopeptide isolated from the yeast cell wall. Biochem. J., 109, 419-432.

SLOMIANY B. L., SLOMIANY A., 1978. Partial characterization of the acidic oligosacharides from rat sublingual glycoproteins. Biochem. biophys. Res. Commun., 81, 391-396.

SPIRO R. G., 1970. Glycoproteins. Annu. Rev. Bioch., 39, 599-638.

TOULlEC R., COROLLER J. Y., PATUREAU-MIRAND P., MELCION J. P., VALDEBOUZE P., DELORT-LAVAL J., CERNING-BEROARD J., 1980. Influence des traitements technologiques sur l'utilisation des protéines de la féverole par le veau préruminant. Ann. Zootech. (à paraître). 